\title{
EVALUACIÓN DE LAS PROPIEDADES TERMODINÁMICAS Y TERMICAS DEL D-LIMONENO ENCAPSULADO MEDIANTE SECADO POR ASPERSIÓN
}

\section{EVALUATION OF THERMODYNAMIC AND THERMAL PROPERTIES FOR D-LIMONENE ENCAPSULATED BY SPRAY DRYING}

\begin{abstract}
Karla Rubiano ${ }^{1}$, Jaime Cárdenas ${ }^{2}$, Héctor J. Ciro-Velásquez ${ }^{3}$
${ }^{1}$ Ingeniera Química, Magister en Ciencia y Tecnología de Alimentos. Universidad Nacional de Colombia, Facultad de Ciencias Agrarias, Departamento de Ingeniería Agrícola y de Alimentos. Calle 39A No. 63-20, Medellín, Colombia, e-mail: kdrubianoc@unal.edu.co; ${ }^{2}$ Ingeniero Químico, M.Sc. TECNAS S.A., Departamento de Innovación. Carrera 50G No. 12Sur-29, Medellín, Colombia, e-mail: jcardenas@tecnas.com.co; ${ }^{3}$ Profesor Asociado, M.Sc., Ph.D. Universidad Nacional de Colombia, Facultad de Ciencias Agrarias, Departamento de Ingeniería Agrícola y de Alimentos. Calle 39A No. 63-20, Medellín, Colombia, e-mail: hjciro@unal.edu.co
\end{abstract}

Rev. U.D.C.A Act. \& Div. Cient. 18(2): 425-434, Julio-Diciembre 2015

\section{RESUMEN}

El objetivo de este estudio fue evaluar las propiedades termodinámicas y térmicas del d-limoneno encapsulado, mediante secado por aspersión, determinando las isotermas de sorción, temperatura de transición vítrea, capacidad calorífica, difusividad y conductividad térmica, complementando la caracterización con análisis termogravimétricos (TGA). Las isotermas de sorción, se establecieron por el método gravimétrico dinámico, a temperaturas de 10,20 y $30^{\circ} \mathrm{C}$, ajustando los datos experimentales a los modelos de BET y GAB, dando como resultado que el modelo de GAB fue el de mejor ajuste. De igual forma, el análisis térmico -para el polvo por calorimetría diferencial de barrido (DSC)-, estableció que la temperatura de transición vítrea es de $97,1^{\circ} \mathrm{C}$ y el análisis termogravimétrico mostró que el producto en polvo presenta una ligera pérdida de volátiles, a $150^{\circ} \mathrm{C}$ y, posteriormente, se produce una degradación térmica, a temperaturas mayores de $200^{\circ} \mathrm{C}$. Asimismo, las propiedades térmicas, tales como la conductividad, difusividad, y el calor específico del producto encapsulado, mostraron los siguientes valores: $0,098 \pm 0,0001 \mathrm{~W} / \mathrm{m}^{\circ} \mathrm{C}, 8,7 \times 10^{-8} \pm 0,0001 \mathrm{~m}^{2} / \mathrm{s}$ y $1,914 \mathrm{~J} / \mathrm{g}^{\circ} \mathrm{C}$ $\pm 0,001$, respectivamente.

Palabras clave: Calor de sorción, temperatura de transición vítrea, análisis termogravimétrico.

\section{SUMMARY}

The objective of this study was to evaluate the thermodynamic and thermal properties of d-limonene, encapsulated by spray drying determining sorption isotherms, glass transition temperature, heat capacity, thermal conductivity and thermal diffusivity, complementing the characterization with thermogravimetric analysis (TGA). Sorption isotherms were determined by dynamic gravimetric method at temperatures of 10,20 and $30^{\circ} \mathrm{C}$ fitting the experimental data to $\mathrm{GAB}$ and BET models, showing that the best fit model was GAB model. Also, thermal analysis for the powder by calorimetry differential scanning (DSC) indicated a value of $97.1^{\circ} \mathrm{C}$ to the glass transition temperature and thermogravimetric analysis showed that the powder presented a slight loss of volatiles at $150^{\circ} \mathrm{C}$ and subsequently thermal degradation occurs after $200^{\circ} \mathrm{C}$. Finally, thermal properties such as conductivity, diffusivity and specific heat of encapsulated product were $0.098 \pm 0.0001 \mathrm{~W} / \mathrm{m}^{\circ} \mathrm{C}, 8.7 \times 10^{-8} \pm 0.0001 \mathrm{~m}^{2} / \mathrm{s}$ and $1.914 \pm 0,0001 \mathrm{~J} / \mathrm{g}^{\circ} \mathrm{C}$, respectively.

Key words: Sorption heat, glass transition temperature, thermogravimetric analysis.

\section{INTRODUCCIÓN}

El d-limoneno es un terpeno que se extrae de la cáscara de frutas cítricas, como el limón y la naranja. Es ampliamente utilizado en la industria farmacéutica, alimentaria y en perfumería, debido a sus propiedades, como aroma y sabor; sin 
embargo, este es un compuesto altamente oxidativo y con elevada sensibilidad a la temperatura, por lo cual, se recurre a técnicas, como la encapsulación, con el fin de proporcionar productos con mayor vida útil y prolongar sus características (Chen et al. 2013).

En los productos alimentarios, conocer la naturaleza fisicoquímica del agua ligada es fundamental, para entender su efecto sobre la estabilidad del producto y las condiciones adecuadas de almacenamiento. Algunas propiedades termodinámicas, como la entalpía, la entropía y la energía libre de Gibbs, pueden ser estimadas a partir de las isotermas de sorción, que expresan la relación que existe entre la actividad de agua y el contenido de humedad del producto, a una temperatura y presión constante (Cano-Higuita et al. 2013). De igual forma, a partir de las isotermas, se puede predecir propiedades higroscópicas de los alimentos: la estabilidad, seleccionar materiales de empaque adecuados y establecer condiciones en cuanto al almacenamiento, de tal forma que no se deteriore el producto (Argyropoulos et al. 2012; Fang et al. 2013).

Adicionalmente, las propiedades termodinámicas son útiles para explicar las reacciones y los fenómenos, a nivel molecular, en los materiales y representan la cantidad de energía, el orden o estado excitado y el equilibrio químico de las moléculas de agua, en el interior del material (Brovchenko \& Oleinikova, 2008). Asimismo, a partir de estas propiedades, se puede estimar el calor de sorción, que es importante en los procesos de adsorción de agua, para productos en polvo y procesos de secado, ya que representa la energía requeri$\mathrm{da}$, para romper las fuerzas intermoleculares entre el vapor de agua y la superficie de producto y se puede utilizar para estimar los requerimientos de energía en procesos de deshidratación y proporciona información importante sobre el estado del agua en los productos (Yan et al. 2008).

Las transiciones de fases en los productos alimentarios conllevan variaciones importantes en las propiedades físicas, que afectan la calidad y la estabilidad del producto. Estas transiciones pueden ser de primer orden, cuando se presentan cambios de la primera derivada de la energía libre, con respecto a la temperatura, como los cambios de estado y desnaturalización o, de segundo orden, cuando se generan cambios en la segunda derivada (Roos, 2010). La temperatura de transición vítrea $(\mathrm{Tg})$ es una transición de segundo orden, que refleja los cambios de un producto de estado amorfo a vítreo, debido a modificaciones en la temperatura o humedad; su conocimiento es también importante para conocer la estabilidad durante el almacenamiento, ya que se recomienda emplear temperaturas de almacenamiento por debajo de la Tg (George et al. 2014).

De igual forma, es importante realizar una caracterización térmica de los productos alimentarios, mediante la deter- minación de propiedades, como la capacidad calorífica, conductividad térmica y difusividad térmica. La capacidad calorífica o calor específico hace referencia a la cantidad de energía requerida para aumentar $1^{\circ} \mathrm{C}$ la temperatura de una muestra y permite la caracterización del estado amorfo, mientras que la conductividad y la difusividad térmica están involucradas en la determinación de la tasa de transferencia de calor, que son útiles en el diseño, la adecuación y la optimización de equipos o de procesos (Sirisomboon \& Posom, 2012). Cabe resaltar que actualmente no se encuentra disponible esta caracterización en productos encapsulados.

El objetivo de este estudio fue evaluar las propiedades termodinámicas, temperatura de transición vítrea, calor específico, difusividad y conductividad térmica para el d-limoneno encapsulado, obtenido mediante secado por aspersión, complementando la caracterización con análisis termogravimétricos (TGA), para cuantificación de pérdida de volátiles y descomposición térmica.

\section{MATERIALES Y MÉTODOS}

Materias primas y localización. El d-limoneno -empleado como ingrediente activo-, la maltodextrina, la goma arábiga, la pectina de bajo metoxilo y tween 60 fueron proporcionados por Tecnas S.A., Medellín, Colombia. El proceso de secado por aspersión, el estudio de isotermas de sorción y las propiedades térmicas del producto fue llevado a cabo en las instalaciones de la empresa Tecnas S.A. y de la Universidad Nacional de Colombia, Sede Medellín.

Proceso de secado por aspersión. El polvo fue obtenido a partir de una emulsión con una composición de $10 \%$ de dlimoneno, 15,9\% de maltodextrina, 15,9\% de goma arábiga, 0,4\% de pectina de bajo metoxilo, $0,1 \%$ de tween 60 y 57,7\% de agua. Para su preparación, se disolvieron los materiales de pared y tensoactivos en agua; luego, se adicionó el d-limoneno y se homogenizó a 12.000rpm, en ULTRA-TURRAX T25, IKA; posteriormente, la emulsión fue secada empleando un secador por aspersión marca Vibrasec S.A., con capacidad de evaporación de $1,5 \mathrm{Lh}^{-1}$, bajo las siguientes condiciones de operación: $156,7^{\circ} \mathrm{C}$, como temperatura del aire de entrada; $90^{\circ} \mathrm{C}$, temperatura del aire de salida y $30.000 \mathrm{rpm}$, como velocidad del disco atomizador.

Caracterización termodinámica. Se determinaron las isotermas de sorción a partir del método gravimétrico estático (Sormoli \& Langrish, 2014), empleando $\mathrm{P}_{2} \mathrm{O}_{5}$ y soluciones salinas saturadas de $\mathrm{CH}_{3} \mathrm{COOK}, \mathrm{MgNO} 3, \mathrm{~K}_{2} \mathrm{CO}_{3}, \mathrm{CuCl}_{2}, \mathrm{NaCl}$ y $\mathrm{KCl}$, especificadas en la tabla 1 . Para ello, se colocaron las soluciones y muestras de $2 \mathrm{~g}$ del encapsulado por triplicado, en recipientes herméticamente cerrados $\mathrm{y}$, posteriormente, se almacenaron a temperaturas de 4,20 y $30^{\circ} \mathrm{C}$, durante 45 días, cuando se alcanzó el equilibrio (Argyropoulos et al. 2012). 
Tabla 1. Humedad relativa del aire en equilibrio, con disoluciones salinas sobresaturadas de algunas sales, a 4,20 y $30^{\circ} \mathrm{C}$ (Martínez et al. 1998).

\begin{tabular}{|c|c|c|c|}
\hline \multirow{2}{*}{ Sal } & \multicolumn{3}{|c|}{ Actividad de agua (aw) } \\
\cline { 2 - 4 } & $4^{\circ} \mathbf{C}$ & $\mathbf{2 0}^{\circ} \mathbf{C}$ & $\mathbf{3 0}^{\circ} \mathbf{C}$ \\
\hline $\mathrm{CH}_{3} \mathrm{COOK}$ & 0,2338 & 0,2311 & 0,2161 \\
\hline $\mathrm{K}_{2} \mathrm{CO}_{3}$ & 0,4314 & 0,4316 & 0,4317 \\
\hline $\mathrm{MgNO}_{3}$ & 0,5736 & 0,5587 & 0,5140 \\
\hline $\mathrm{CuCl}_{2}$ & 0,6800 & 0,6800 & 0,6750 \\
\hline $\mathrm{NaCl}$ & 0,7565 & 0,7547 & 0,7509 \\
\hline $\mathrm{KCl}$ & 0,8677 & 0,8511 & 0,8362 \\
\hline
\end{tabular}

Los datos de actividad de agua, de temperatura y de contenido de humedad de equilibrio, obtenidos experimentalmente, fueron ajustados a los modelos de BET, ecuación 1 y GAB, ecuación 2, citados por Andrade et al. (2011):

$$
\begin{aligned}
& X_{w}=\frac{X_{m} C a_{w}}{\left(1-a_{w}\right)\left(1+(C-1) a_{w}\right)} \\
& X_{w}=\frac{X_{m} C K a_{w}}{\left(1-K a_{w}\right)\left(1+(C-1) K a_{w}\right)}
\end{aligned}
$$

$\mathrm{X}_{\mathrm{w}}$ es el contenido de humedad en el equilibrio; $\mathrm{X}_{\mathrm{m}}$ es el contenido de humedad en la monocapa; $a_{w}$ es la actividad de agua y, C y K son constantes para los modelos.

Se determinó la bondad de ajuste de cada modelo a los datos experimentales, mediante el coeficiente de determinación $\left(\mathrm{R}^{2}\right)$, menor media cuadrada de la desviación $\left(\mathrm{X}^{2}\right)$ y la raíz cuadrada del error medio (ERMS). Para un buen ajuste, se eligió el mayor valor de $\mathrm{R}^{2}$ y los menores valores de $\mathrm{X}^{2}$ y ERMS (Goyal et al. 2006; Bingol et al. 2012):

$$
\begin{aligned}
& X^{2}=\frac{\sum_{i=1}^{N}\left(M_{R, \text { exp }, i}-M_{R, p r e, i}\right)^{2}}{N-z} \\
& E_{R M S}=\left[\frac{1}{N} \sum_{i=1}^{N}\left(M_{R, \exp , i}-M_{R, p r e, i}\right)^{2}\right]^{1 / 2}
\end{aligned}
$$

MR,exp,i y MR,pre,i son los datos experimentales y calculados para cada modelo de la humedad de equilibrio (Xw), respectivamente; $N$ es el número de observaciones $\mathrm{y}$, $\mathrm{z}$ es el número de constantes de cada modelo.

La ecuación de Clausius-Clapeiron, para el equilibrio líquidovapor, se aplicó a los datos experimentales de temperatura y de presión de vapor del producto, con el fin de calcular el cambio en la entalpía para diferentes niveles de humedad, asociados con el proceso de sorción, es decir, el calor diferencial de adsorción (Yan et al. 2008; Sormoli \& Langrish, 2014).

$$
\begin{aligned}
& \ln P=\frac{-\Delta H}{R} \frac{1}{T}+C t e \\
& P=P_{w}^{0} a_{w}
\end{aligned}
$$

Donde, $\mathrm{P}$ presión de vapor del agua en equilibrio con el producto, a una temperatura determinada en atm; $\Delta \mathrm{H}$ es el calor isostérico de sorción $\mathrm{kJ} / \mathrm{mol}$; $\mathrm{R}$ constante universal de los gases $8,314 \times 10^{-3} \mathrm{~kJ} / \mathrm{mol}$; T es la temperatura de adsorción $\mathrm{K} ; \mathrm{P}_{\mathrm{w}}{ }^{0}$ en atm es la presión de vapor del agua pura y aw es la actividad de agua, a una temperatura determinada. Luego, se determinó el calor isostérico neto de sorción $Q_{s}$, mediante la ecuación 7, que aporta información importante acerca de los cambios energéticos que ocurren en los alimentos, a un nivel de hidratación específico, producidos por cambios diferenciales en la humedad de equilibrio del producto (Viganó et al. 2012); adicionalmente, se estableció la energía libre de Gibbs, con la ecuación 8 y la entropía de adsorción, con la ecuación 9:

$$
\begin{aligned}
& \Delta H=\Delta H_{V}+Q_{S} \\
& \Delta \mathrm{G}=R T \ln \left(\frac{P}{P^{0}}\right)=R T \ln \left(a_{w}\right) \\
& \Delta \mathrm{S}=\frac{\Delta H-\Delta G}{T}
\end{aligned}
$$

Donde, $\Delta \mathrm{H}$ es el calor isostérico de sorción $\mathrm{kJ} / \mathrm{mol} ; \Delta \mathrm{Hv}$, el calor de evaporación del agua $\mathrm{kJ} / \mathrm{mol}$; Qs, calor neto de sorción $\mathrm{kJ} / \mathrm{mol} ; \Delta \mathrm{G}$, energía libre de Gibbs $\mathrm{kJ} / \mathrm{mol}$ y $\Delta \mathrm{S}$, entropía de adsorción kJ/molK.

Transición de fases. La temperatura de transición vítrea fue determinada por triplicado, con un calorímetro diferencial de barrido Q2000, TA Instruments, para lo cual, se tomaron 10mg de muestra en un crisol de aluminio, herméticamente sellado. La velocidad de calentamiento y de enfriamiento 
modulado, se fijó a $2^{\circ} \mathrm{C} / \mathrm{min}$, en un rango de temperatura de $-10^{\circ} \mathrm{C}$ a $200^{\circ} \mathrm{C}$, con amplitud de $1,3^{\circ} \mathrm{C}$ y periodo de oscilación de 60s (Chen et al. 2013).

La estabilidad térmica del d-limoneno encapsulado, se evaluó mediante análisis termogravimétrico (TGA), en una termobalanza Linseis STA PT1600, de acuerdo a la metodología con algunas modificaciones dada por Wen et al. (2014). Se empleó una atmosfera de nitrógeno con flujo de $50 \mathrm{~mL} /$ min, velocidad de calentamiento de $10^{\circ} \mathrm{C} / \mathrm{min}$, en un intervalo de temperatura de 20 a $300^{\circ} \mathrm{C}$. Se pesaron $10 \mathrm{mg}$ de muestra en crisol de aluminio realizándose las determinaciones por duplicado.

Propiedades térmicas. Se determinaron la conductividad (k) y la difusividad térmica $(\alpha)$ del d-limoneno encapsulado, mediante el equipo KD2 Pro, Decagon Devices, Pullman, WA, USA, con la sonda SH-1, el cual, es un sensor de doble aguja con $1,3 \mathrm{~mm}$ de diámetro, $3 \mathrm{~cm}$ de largo y $6 \mathrm{~mm}$ de distancia, entre ellas. Este sensor contiene un elemento de calentamiento y una resistencia térmica, por lo que se insertó verticalmente en la muestra, para asegurar que la convección libre se reduzca al mínimo. Este equipo emplea el método de calor de línea transitoria, para medir la conductividad y la difusividad térmica. Se tomaron cinco mediciones, con un intervalo de $15 \mathrm{~min}$, entre cada una, para asegurar la reproducibilidad (França et al. 2014); posteriormente, se estableció la densidad de producto, mediante el método del picnómetro y se calculó la capacidad calorífica, de forma indirecta, a partir de la ecuación 10. Este resultado fue comparado con el obtenido, mediante el análisis por calorimetría diferencial de barrido (DSC):

$$
\mathrm{Cp}=\frac{k}{\rho \alpha}
$$

Donde, $\kappa$ es la conductividad $(\mathrm{W} / \mathrm{mK}) ; \rho$ es la densidad $(\mathrm{kg} /$ $\left.\mathrm{m}^{3}\right) \mathrm{y}, \alpha$ es la difusividad térmica $\left(\mathrm{m}^{2} / \mathrm{s}\right)$ del d-limoneno encapsulado.

\section{RESULTADOS Y DISCUSIÓN}

En la figura 1, se presenta la relación que existe entre la actividad de agua y el contenido de humedad de equilibrio, es decir, las isotermas de sorción, para el d-limoneno encapsulado. Las curvas describen una forma sigmoidea tipo II, las cuales, son típicas en productos alimentarios; se caracterizan porque el producto adsorbe pequeñas cantidades de agua a valores bajos de actividad de agua, seguido por una adsorción gradual de humedad al aumentar la actividad de agua, de acuerdo con lo reportado por Argyropoulos et al. 2012. También, se observa -a partir de los resultados-, que existe una relación entre el incremento de la temperatura y la disminución en el contenido de humedad, por lo cual, la hi- groscopicidad del encapsulado disminuye con el incremento de la temperatura; resultados similares han sido reportados por Fang et al. (2013), para el d-limoneno encapsulado con $\beta$-ciclodextrina y Cano-Higuita et al. (2013), en mango en polvo que contenía maltodextrina y leche desnatada.

En la tabla 2, se registran los parámetros para los modelos de GAB y BET y los respectivos coeficientes de determinación, la menor media cuadrada de la desviación y el error medio para cada modelo. Se observa que el modelo de GAB mostró un mejor ajuste a los datos experimentales, con mayores coeficientes de determinación para todas las temperaturas analizadas y los menores valores de $\mathrm{X}^{2}$ y ERMS; aunque para $30^{\circ} \mathrm{C}$ estos fueron ligeramente mayores, posiblemente, a variaciones aleatorias en la cámara climática empleada.

La humedad de la monocapa $\left(\mathrm{X}_{\mathrm{m}}\right)$, que establece la cantidad de agua que se encuentra fuertemente ligada al alimento, disminuyó con el aumento de la temperatura para ambos modelos, lo que se puede deber a la menor disponibilidad de agua en las uniones puntos activos $-\mathrm{H}_{2} \mathrm{O}$ o $\mathrm{H}_{2} \mathrm{O}-\mathrm{H}_{2} \mathrm{O}$ (Westgate et al. 1992). Asimismo, un aumento de la temperatura provoca una mayor activación de las moléculas de agua, debido al aumento en el nivel de energía, favoreciendo, de este modo, el rompimiento de los enlaces moleculares de agua y, por lo tanto, una disminución en el contenido de humedad de la monocapa. Para el modelo de GAB, el de mejor ajuste, varió entre 2,69 y 9,2\%. Resultados similares fueron reportados por Fang et al. (2013), al emplear 9,68\% de limoneno.

La constante $\mathrm{C}$ relaciona el calor de sorción de la primera capa en los sitios primarios de la muestra y presentó valores entre 2,80 y 5,48 , similares a los reportados por Cano-Higuita et al. (2013), reflejando un aumento con la temperatura; similarmente, el valor $\mathrm{K}$, que proporciona una medida de la interacción de las moléculas en las multicapas con el producto, registró valores entre 0,87-1,01 y también aumentó con el incremento de la temperatura. Según lo anterior, a una mayor temperatura aumentan las interacciones entre el agua y el alimento, causando un incremento en la constante C y K, de acuerdo con lo reportado por Lavoyer et al. (2013). Los resultados obtenidos para el modelo de GAB coinciden con otros estudios realizados en aceites esenciales encapsulados y jugo de naranja en polvo (Fernandes et al. 2014; Sormoli \& Langrish, 2014).

En la figura 2, se explica la caracterización termodinámica para el d-limoneno encapsulado, a partir del calor isostérico de sorción, energía libre de Gibbs y entropía, que fueron calculados con el modelo de GAB y las ecuaciones descritas previamente.

El calor isostérico neto de sorción para el d-limoneno encapsulado varió entre 15,17 y $20,82 \mathrm{~kJ} / \mathrm{mol}$, presentando un au- 


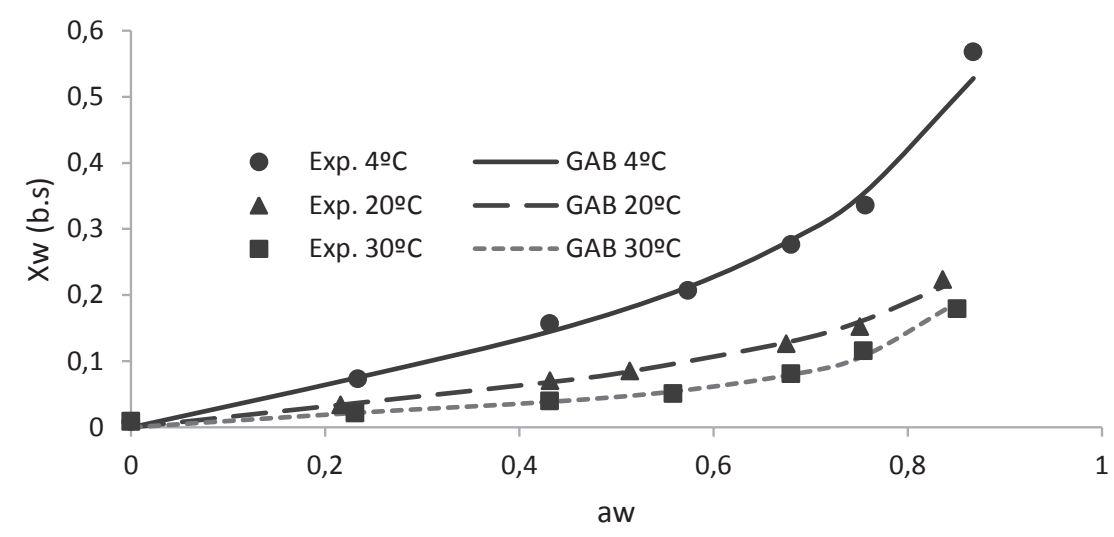

A

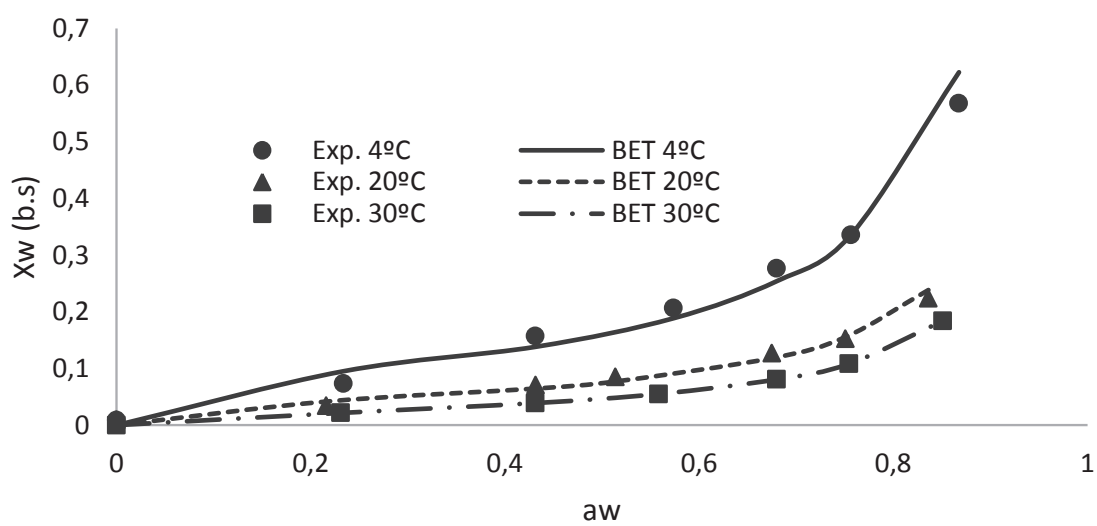

B

Figura 1. Isotermas de sorción para el d-limoneno encapsulado bajo el modelo de GAB (A) y BET (B).

Tabla 2. Parámetros para el modelo de GAB y BET, a diferentes temperaturas.

\begin{tabular}{|c|c|c|c|c|}
\hline MODELO & PARÁMETROS & $4^{\circ} \mathrm{C}$ & $20^{\circ} \mathrm{C}$ & $30^{\circ} \mathrm{C}$ \\
\hline \multirow{4}{*}{ GAB } & $\mathbf{X}_{\mathbf{m}}$ & 0,092 & 0,0641 & 0,0269 \\
\cline { 2 - 5 } & $\mathbf{C}$ & 2,8037 & 3,3027 & 5,4873 \\
\cline { 2 - 5 } & $\mathbf{k}$ & 0,8702 & 0,8716 & 1,0127 \\
\cline { 2 - 5 } & $\mathbf{R}^{2}$ & 0,9387 & 0,9722 & 0,9748 \\
\cline { 2 - 5 } & $\mathbf{X}^{2}$ & 0,0008 & $9,36 \times 10^{-5}$ & $8,43 \times 10^{-5}$ \\
\hline \multirow{4}{*}{ BET } & $\mathrm{E}_{\text {RMS }}$ & 0,0197 & 0,0068 & 0,0065 \\
\cline { 2 - 5 } & $\mathbf{X}_{\mathbf{m}}$ & 0,0829 & 0,0395 & 0,0284 \\
\cline { 2 - 5 } & $\mathbf{C}$ & 23,9952 & 17,3937 & 4,6553 \\
\cline { 2 - 5 } & $\mathbf{R}^{2}$ & 0,9174 & 0,9472 & 0,9644 \\
\cline { 2 - 5 } & $\mathbf{X}^{2}$ & 0,0012 & 0,00013 & $4,48 \times 10^{-5}$ \\
\hline
\end{tabular}



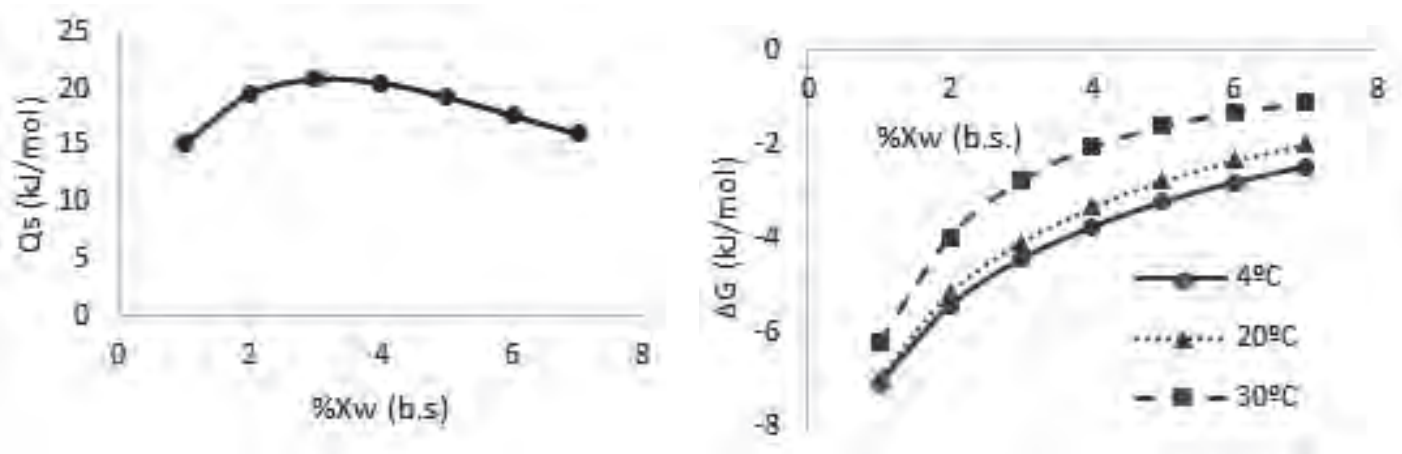

A

B

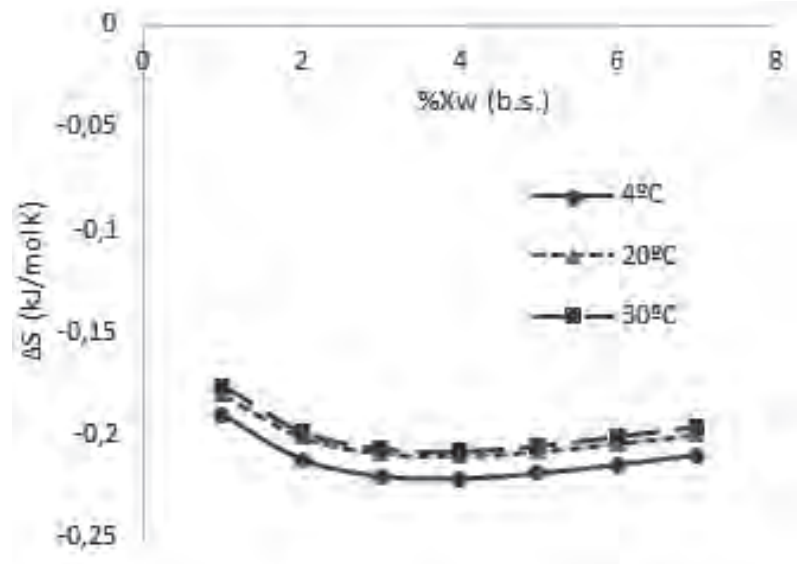

C

Figura 2. Caracterización termodinámica para el d-limoneno encapsulado: (A) Calor isostérico neto de sorción (Qs); (B) cambio de la energía libre de Gibbs (DG) y (C) cambio de la entropía $(\Delta S)$.

mento con bajos contenidos de humedad hasta 3\% y, luego, tiende a disminuir, con una humedad mayor de $4 \%$ (Figura 2A); comportamientos similares han sido reportados para el calor isosterico neto de polvo de naranja (Sormoli \& Langrish, 2014).Cabe resaltar, que el contenido de humedad, donde el calor de sorción es máximo, se ajusta aproximadamente al valor de la monocapa, que representa la humedad del producto, cuando los sitios de adsorción primarios están saturados por moléculas de agua, lo que ocasiona que el vapor de agua sea adsorbido en zonas menos accesibles, por lo que el calor de sorción es mayor, justo antes de la finalización de la monocapa (Martínez et al. 2014). El decrecimiento progresivo del calor de sorción con el incremento del contenido de humedad, se debe a que en el proceso inicial se produce en los sitios disponibles más activos, dando lugar a una alta energía de interacción y, a medida que estos sitios de forma progresiva se ocupan, la sorción se produce en los sitios menos activos, dando lugar a un menor requerimiento de energía.
La energía libre de Gibbs relaciona el trabajo necesario para que el producto tenga sitios disponibles para la sorción. Los resultados obtenidos indican que la adsorción de agua -para el d-limoneno encapsulado- es un proceso espontáneo, ya que se obtuvieron valores negativos $(\mathrm{DG}<0)$, que es característico de fenómenos de adsorción. De la figura 2B, se observa que la energía libre de Gibbs tiende a cero, cuando el contenido de humedad es mayor, ya que habrá menos sitios disponibles para la sorción, con altos contenidos de humedad. Asimismo, la energía libre de Gibbs disminuye al aumentar la temperatura, debido a que cuando se incrementa esta variable la vibración de las moléculas aumenta, requiriéndose menos trabajo, para que hayan sitios disponibles para la sorción (Goneli et al. 2013). Por lo tanto y de acuerdo con los resultados, el d-limoneno encapsulado será más higroscópico si se almacena a una baja temperatura; resultados similares fueron encontrados para mango y para piña en polvo (Viganó et al. 2012; Cano-Higuita et al. 2013). 
El cambio en la entropía es proporcional al número de sitios de adsorción, disponibles en un nivel específico de energía y está asociado con las fuerzas de atracción o repulsión, presentada por la interfase del agua y el absorbente (Ceballos et al. 2013). La figura 2C muestra una tendencia a disminuir $\Delta \mathrm{S}$ ligeramente, con un aumento del contenido de humedad, tendencia que puede ser explicada por el hecho que un aumento del contenido de humedad implica menos sitios de unión disponibles y, por lo tanto, una disminución de la entropía.

Adicionalmente, se observa que el cambio de entropía diferencial $(\Delta S)$ es menor con la disminución de la temperatura. Este comportamiento, se debe a que la energía cinética de las moléculas, que actúan en el intercambio de agua, es menor al disminuir la temperatura y, por lo tanto, tienen menor movilidad. Los resultados para el d-limoneno encapsulado corresponden a valores negativos, que se puede atribuir a la existencia de adsorción química y modificaciones estructurales en el adsorbente, que ocasiona una pérdida de movilidad de las moléculas de agua, durante la sorción (Xiao \& Tong, 2013).

La figura 3 muestra el termograma obtenido por DSC, para el d-limoneno encapsulado, donde se observa que la temperatura de transición vítrea es aproximadamente $97,1^{\circ} \mathrm{C}$, comenzando en $93,1^{\circ} \mathrm{C}$ y con un punto final, en $107,5^{\circ} \mathrm{C}$. El alto valor en la temperatura de transición vítrea es debido al bajo contenido de humedad del producto, lo que, a su vez, evita problemas de apelmazamiento y de plastificación (Subramaniam et al. 2013). También, se observa que alrededor de $150^{\circ} \mathrm{C}$ se genera un pico endotérmico en el flujo de calor total y debido a que este pico coincide con la señal del flujo de calor no reversible, representa una pérdida de algún componente volátil en ese rango de temperatura, que podría ser el d-limoneno.

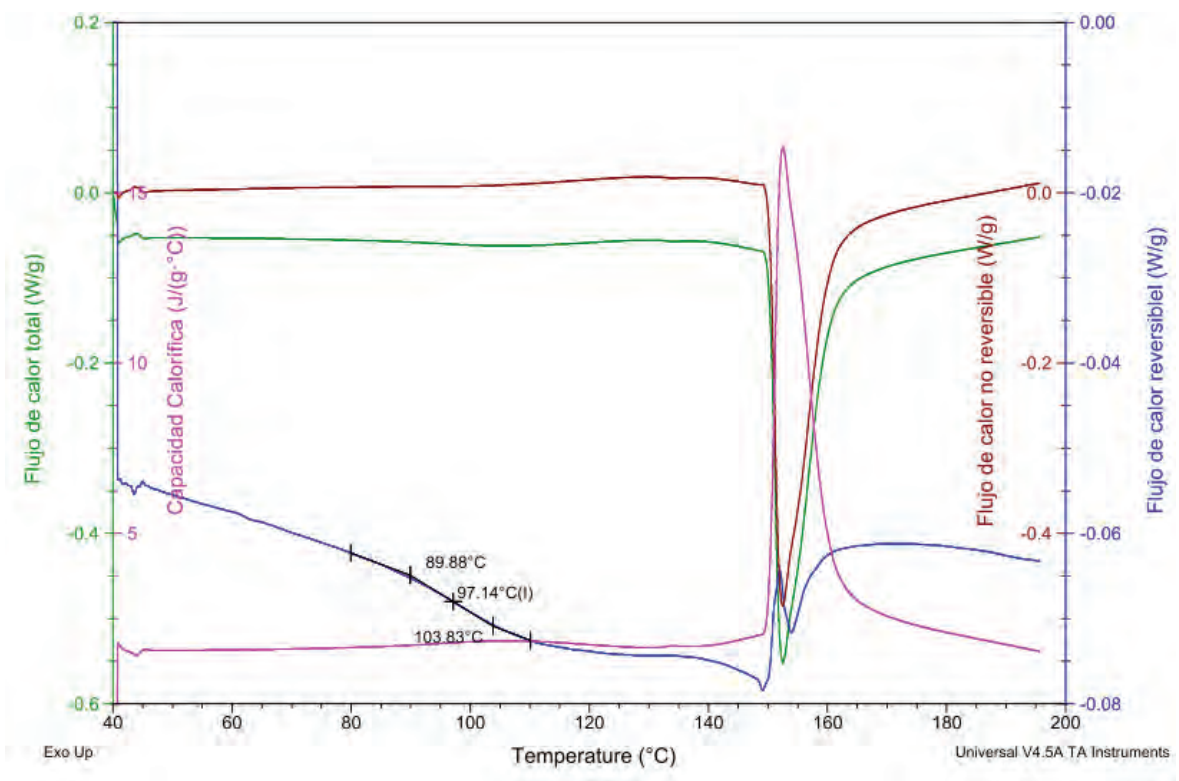

Figura 3. Análisis térmico con DSC para el d-limoneno encapsulado, mediante secado por aspersión.

La maltodextrina y goma arábiga, componentes de mayor proporción en el polvo, tienen temperaturas de transición vítrea de $162^{\circ} \mathrm{C}$ y $280^{\circ} \mathrm{C}$, respectivamente (Kurozawa et al. 2009), mientras que en el producto encapsulado, se presenta una disminución, debido a la presencia de componentes de bajo peso molecular, como el d-limoneno. De igual forma, la temperatura de transición vítrea, obtenida para el producto, garantiza su estabilidad durante el almacenamiento, puesto que las temperaturas son menores de $40^{\circ} \mathrm{C} \mathrm{y}$, a su vez, en el proceso de secado por aspersión, donde la temperatura de salida fue $90^{\circ} \mathrm{C}$.

De las curvas termogravimétricas (TGA), para dos muestras del producto en polvo (Figura 4), se puede observar que el d-limoneno encapsulado presenta una buena estabilidad térmica, a temperaturas menores de $150^{\circ} \mathrm{C}$, ya que no hay pérdida de masa o volátiles significativa, lo que indica la estabilidad de las microcápsulas, en ese rango de temperatura; 
sin embargo, al aumentar la temperatura, se observa una leve pérdida de masa, que corresponde a pérdida de volátiles, que coinciden con los resultados reportados mediante el DSC en el flujo de calor no reversible; posteriormente, por encima de $200^{\circ} \mathrm{C}$, las microcápsulas se van descomponiendo y pierden casi todo su masa, por encima de $300^{\circ} \mathrm{C}$; esta pérdida, se puede atribuir a la degradación de los polisacáridos presentes en el producto encapsulado, como goma arábiga y maltodextrina, que se emplearon como materiales de pared en la encapsulación, ya que se ha encontrado en la literatura que la descomposición térmica de los polisacáridos se encuentran en esta región e, incluye, varios procesos, como la deshidratación, la despolimerización y la formación de las estructuras de grafito y de carbonos aromáticos (Wen et al. 2014). De igual forma, los resultados encontrados concuerdan con Levic et al. (2011), quienes hicieron un análisis termográvimétrico al d-limoneno encapsulado con alginato y polivinil alcohol, encontrando que no hay una pérdida de masa significativa, hasta $200^{\circ} \mathrm{C}$ y evidenciando la estabilización térmica con la encapsulación.
Los resultados del análisis de propiedades térmicas -en un rango de temperatura de 25 a $40^{\circ} \mathrm{C}$, mostraron que el dlimoneno encapsulado tiene una conductividad térmica de $0,098 \pm 0,0001 \mathrm{~W} / \mathrm{mK}$ y difusividad térmica de $8,7 \times 10^{-}$ ${ }^{8} \pm 0,0001 \mathrm{~m}^{2} / \mathrm{s}$. Se puede observar que el valor de la conductividad es bajo, debido a la baja humedad del producto. De acuerdo con Aviara et al. (2008), la conductividad aumenta al incrementar la humedad y la temperatura. De igual forma, los resultados encontrados para la difusividad y la conductividad térmica son similares a los reportados por otras investigaciones, en mediciones de propiedades térmicas de semillas (Aviara et al. 2008; Sirisomboon \& Posom, 2012) y de harina de trigo (Huang \& Liu, 2009), pero para matrices formuladas con d-limoneno encapsulado, no han sido reportadas. Por otro lado, la densidad del polvo, calculada mediante el método del picnómetro, fue de $582,683 \mathrm{~kg} /$ $\mathrm{m}^{3} \pm 0,15$, con una humedad del $2,22 \%$, en base húmeda $\mathrm{y}$, con estos resultados, fue utilizada la ecuación 8 , obteniéndose un calor específico de $1,914 \mathrm{~J} / \mathrm{gK}$, mientras que para el análisis con DSC, se registró un valor 1,791J/gK, que son

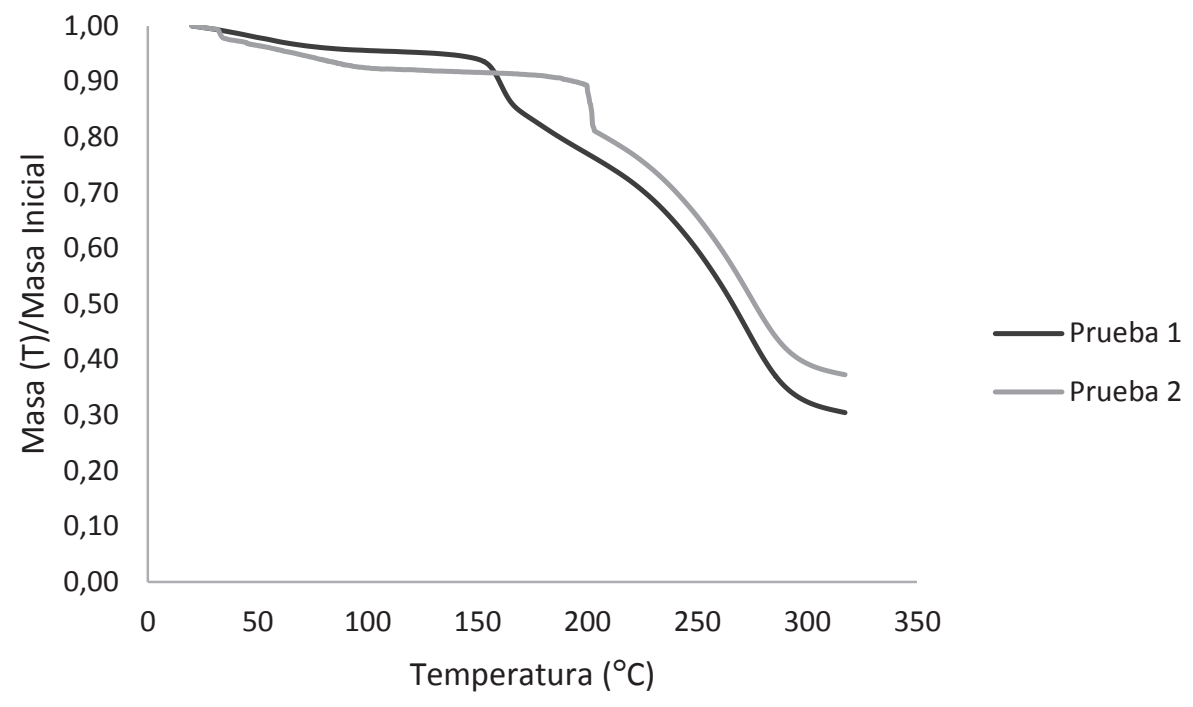

Figura 4. Análisis termogravimétrico para el d-limoneno encapsulado, mediante secado por aspersión, realizado a dos muestras bajo condiciones similares, especificadas como prueba 1 y 2 .

muy próximos; sin embargo, si se aplica la ecuación de Siebel para calcularla (Onita \& Ivan, 2005), se obtiene un valor de $0,912 \mathrm{~J} / \mathrm{gK}$, que son diferentes, debido a que esta última aproximación es más aplicable y valida a sólidos secos de frutas libres de grasa.

Se concluye, que la isoterma de sorción para el d-limoneno presenta una forma sigmoidea tipo II y el modelo de GAB mostró un mejor ajuste en el modelamiento de los datos experimentales, en el intervalo de actividad de agua, entre $0 \mathrm{y}$ 0,836 , a temperaturas de 4,20 y $30^{\circ} \mathrm{C}$. También, se evidencia que el d-limoneno encapsulado es estable a las condiciones de almacenamiento, ya que posee una alta temperatura de transición vítrea $(97,1$ 으) y, a su vez, es estable térmicamente hasta $150^{\circ} \mathrm{C}$, debido a la protección de los materiales de cobertura empleados. Las propiedades térmicas para el 
d-limoneno encapsulado fueron conductividad térmica de $0,098 \mathrm{~W} / \mathrm{mK}$, difusividad de $8,7 \times 10^{-8} \mathrm{~m}^{2} / \mathrm{s}$ y calor especifico $1,914 \mathrm{~J} / \mathrm{gK}$, reflejando que el producto en polvo es estable, debido a su baja conductividad térmica, relacionada con el bajo contenido de humedad y es estable en las condiciones de almacenamiento.

Agradecimientos. Los autores expresan sus agradecimientos a la Dirección de Investigaciones Sede Medellín (DIME) de la Universidad Nacional de Colombia, a Colciencias y a la empresa Tecnas S.A., por el apoyo financiero y de infraestructura recibido. Conflictos de intereses: El artículo fue preparado y revisado con la participación de todos los autores, quienes declaramos que no existe conflicto de intereses que ponga en riesgo la validez de los resultados presentados. Financiación: Este estudio fue financiado por Tecnas S.A., Colciencias y la Universidad Nacional de Colombia, Sede Medellín.

\section{BIBLIOGRAFÍA}

1. ARGYROPOULOS, D.; ALEX, R.; KOHLER, R.; MÜLLER, J. 2012. Moisture sorption isotherms and isosteric heat of sorption of leaves and stems of lemon balm (Melissa officinalis L.) established by dynamic vapor sorption. LWT - Food Sci. Techn. (Estados Unidos). 47(2):324-331.

2. ANDRADE, R.; LEMUS, R.; PÉREZ, C. 2011. Models of sorption isotherms for food: uses and limitations. VITAE, Rev. Fac. Química Farmacéutica (Colombia). 18(3):325-334.

3. AVIARA, N.; HAQUE, M.; OGUNJIMI, L. 2008. Thermal properties of guna seed. Int. Agrophysics (Polonia). 22:291-297.

4. BINGOL, G.; PRAKASH, B.; PAN, Z. 2012. Dynamic vapor sorption isotherms of medium grain rice varieties. LWT - Food Sci. Techn. 48(2):156-163.

5. BROVCHENKO, I.; OLEINIKOVA, A. 2008. Interfacial and Confined Water. Ed. Elsevier (Inglaterra). 305p.

6. CANO-HIGUITA, D.; VILLA, H.; TELIS, J.; VÁQUIRO, H.; NICOLETTI, V. 2013. Influence of alternative drying aids on water sorption of spray dried mango mix powders: A thermodynamic approach. Food Biopr. Process. (Reino Unido). 93:19-28.

7. CEBALlOS, A.; GIRALDO, G.; ORREGO, C.; TELIS, J. 2013. Sorption thermodynamics of soursop powders obtained by different dehydration technologies. Rev. Fac. Nal. Agr.-Medellín. 66(2):7107-7116.
8. CHEN, Q.; MCGILLIVRAY, D.; WEN, J.; ZHONG, F.; QUEK, S. 2013. Co-encapsulation of fish oil with phytosterol esters and limonene by milk proteins. J. Food Eng. 117(4):505-512.

9. FANG, Z.; COMINO, P.; BHANDARI, B. 2013. Effect of encapsulation of $D$-limonene on the moisture adsorption property of b -cyclodextrin. LWT - Food Sci. Techn. 51(1):164-169.

10. FERNANDES, R.; BORGES, S.; BOTREL, D. 2014. Gum arabic/starch/maltodextrin/inulin as wall materials on the microencapsulation of rosemary essential oil. Carbohydr. Polymers (Reino Unido). 101:524- 532.

11. FRANÇA, J.; REIS, F.; VIEIRA, S.; LOURENÇO, M.; SANTOS, F.; NIETO DE CASTRO, C.; PÁDUA, A. 2014. Thermophysical properties of ionic liquid dicyanamide (DCA) nanosystems. J. Chem. Thermodyn. (Estados (Unidos). 79:248-257.

12. GEORGE, P.; LUNDIN, L.; KASAPIS, S. 2014. Effect of thermal denaturation on the mechanical glass transition temperature of globular protein/co-solute systems. Food Hydrocoll. 41:156-163.

13. GONELI, A.; CORRÉA, P.; OLIVEIRA, G.; AFONDO, P. 2013. Water sorption properties of coffee fruits, pulped and green coffee. LWT - Food Sci. Techn. 50(2):386-391.

14. GOYAL, R.; KINGSLY, A.; MANIKANTAN, M.; ILYAS, S. 2006. Thin-layer Drying Kinetics of Raw Mango Slices. Biosystems Eng. (Estados Unidos). 95(1):4349.

15. HUANG, L.; LIU, L. 2009. Simultaneous determination of thermal conductivity and thermal diffusivity of food and agricultural materials using a transient plane-source method. J. Food Eng. 95(1):179-185.

16. KUROZAWA, L.; PARK, K.; HUBINGER, M. 2009. Effect of maltodextrin and gum arabic on water sorption and glass transition temperature of spray dried chicken meat hydrolysate protein. J. Food Eng. 91(2):287296.

17. LAVOYER, F.; GABAS, A.; OLIVEIRA, W.; TELIS, J. 2013. Study of adsorption isotherms of green coconut pulp. Ciênc. Tecn. Alimentos, Campinas (Brasil). 33(1):68-74.

18. LEVIC, S.; RAC, V.; MANOJLOVI, V.; RAKI, V.; BUGARSKI, B.; FLOCK, T.; KRZYCZMONIK, K.; NEDOVI, V. 
2011. Limonene encapsulation in alginate/poly (vinyl alcohol). Procedia Food Sci. (Inglaterra). 1:18161820.

19. MARTÍNEZ, R.; HEREDIA, A.; CASTELLÓ, M.; ANDRÉS, A. 2014. Moisture sorption isotherms and isosteric heat of sorption of dry persimmon leaves. Food Biosci. (Reino Unido). 7:88-94.

20. MARTÍNEZ, N.; ANDRÉS, A.; CHIRALT, A.; FITO, P. 1998. Termodinámica y Cinética de Sistemas: Alimento y Entorno. Ed. Universidad Politécnica de Valencia (España). 372p.

21. ONITA, N.; IVAN, E. 2005. Estimation of the specific heat and thermal conductivity of foods only by their classes of substances contents (water, proteins, fats, carbohydrates, fibers and ash). Agroalimen. Process. Techn. (Romania). 10(1):217-222.

22. ROOS, Y. 2010. Glass transition temperature and its relevance in food processing. Ann. Rev. Food Sci. Techn. (Estados Unidos). 1:469-496.

23. SIRISOMBOON, P.; POSOM, J. 2012. Thermal properties of Jatropha curcas L. kernels. Biosystems Engineering (Estados Unidos). 113(4):402-409.

24. SORMOLI, M.; LANGRISH, T. 2014. Moisture sorption isotherms and net isosteric heat of sorption for spray-dried pure orange juice powder. LWT Food Sci. Techn. Disponible desde Internet en: http:// dx.doi.org/10.1016/j.lwt.2014.09.064 (con acceso 10/11/2014).
25. SUBRAMANIAM, A.; VEAZEY, R.; SCHOBER, A.; RADA, A.; RONG, Y.; SLEEUWEN, R.; GOLDING, R.; ZHANG, S.; NORMAND, V. 2013. Orange oil stability in spray dry delivery systems. Carbohydr. Polymers. 97(2):352-357.

26. VIGANÓ, J.; AZUARA, E.; TELIS, V.; BERISTAIN, C.; JIMÉNEZ, M.; TELIS, T. 2012. Role of enthalpy and entropy in moisture sorption behavior of pineapple pulp powder produced by different drying methods. Thermochimica Acta (Holanda). 528:63-71.

27. WEN, CH.; YUAN, Q.; LIANG, Q.; VRIESEKOOP, F. 2014. Preparation and stabilization of d-limonene Pickering emulsions by cellulose nanocrystals. Carbohydr. Polymers. 112:695-700.

28. WESTGATE, N.; LEE, J.; LADISCH, M. 1992. Modelling Of Equilibrium Sorption of Water Vapour on Starchy Materials. Am. Soc. Agr. Eng. (Estados Unidos). 35:213-219.

29. XIAO, Q.; TONG, Q. 2013. Thermodynamic properties of moisture sorption in pullulan-sodium alginate based edible films. Food Res. Int. 54(2):1605-1612.

30. YAN, Z.; SOUSA, M.; OLIVEIRA, F. 2008. Sorption isotherms and moisture sorption hysteresis of intermediate moisture content banana. J. Food Eng. 86(3):342-348.

Recibido: Febrero 10 de 2015

Aceptado: Julio 1 de 2015

\section{Cómo citar:}

Rubiano, K.; Cárdenas, J.; Ciro-Velásquez, H.J. 2015. Evaluación de las propiedades termodinámicas y térmicas del d-limoneno encapsulado mediante secado por aspersión. Rev. U.D.C.A Act. \& Div. Cient. 18(2): 425-434. 